\title{
Las prácticas de enseñanza empleadas por docentes de matemáticas y su relación con la resolución de problemas mediados por fracciones ${ }^{1}$
}

\author{
Práticas pedagógicas utilizadas pelos professores de matemática \\ e sua relação com a resolução de problemas frações mediada
}

Práticas pedagógicas utilizadas pelos professores de matemática e sua relação com a resolução de problemas frações mediada

Recibido: mayo de 2013

Aprobado: agosto de 2013
Alexander Murillo Moreno ${ }^{2}$

Leonardo Ceballos Urrego ${ }^{3}$

\section{Resumen}

La enseñanza a través de resolución de problemas enfatiza en procesos de pensamiento y aprendizaje, por ello, es el método en que actualmente se apoyan los docentes para poner en práctica el aprendizaje activo. Lo que se persigue, es transmitir de manera sistemática, procesos de pensamiento eficaces en resolución de problemas. Este trabajo se enmarca en una metodología de investigación cualitativa, cuyo diseño metodológico es estudio de caso colectivo, y parte de una relación histórica frente a teorías y aportes significativos, asociados con las prácticas empleadas por docentes de matemáticas para la enseñanza, la heurística y métodos para la resolución de problemas mediados por fracciones, indagando hasta que punto dichas prácticas favorecen las habilidades de los estudiantes.

Palabras clave: Prácticas de enseñanza; docentes; otras nociones de Educación Matemática; resolución de problemas; matemáticas escolares; números; estructuras numéricas; números racionales; fracciones.

\begin{abstract}
Teaching through problem solving emphasizes thinking and learning processes, therefore, is the method currently support teachers to implement active learning. What is pursued is transmitted systematically effective thought processes in problem solving. This work is part of a qualitative research methodology, the methodological design collective case study, and part of a historical relationship against theories and significant contributions associated with the practices used by teachers for teaching mathematics, heuristics and methods for mediated problem solving fractions, investigating to what extent these practices support students skills.
\end{abstract}

$1 \quad$ Artículo de Investigación

2 Universidad de Antioquia, Medellin, Colombia. Contacto: alexanderm54@gmail.com

3 Universidad de Antioquia, Medellin, Colombia. Contacto: lceu0457@gmail.com 
Palabras clave: Teaching practices, teachers, other notions of mathematics education, problem solving, school mathematics, numbers, numerical structures, rational numbers, fractions.

\section{Resumo}

Ensinar através de resolução de problemas enfatiza processos de pensamento e aprendizagem, portanto, é o método atualmente em apoiar os professores para implementar o aprendizado ativo. O que é perseguido é transmitida processos de pensamento sistematicamente eficazes na resolução de problemas. Este trabalho faz parte de uma metodologia de pesquisa qualitativa, o estudo de caso coletivo desenho metodológico, e parte de uma relação histórica contra as teorias e contribuições significativas associadas com as práticas utilizadas pelos professores para o ensino da matemática, a heurística e métodos para resolver problema mediada frações, investigando em que medida essas práticas apoiar as habilidades dos estudantes.

Palabras clave: Práticas de ensino, professores, outras noções de educação matemática, resolução de problemas, a matemática da escola, números, estruturas numéricas, números racionais e frações

\section{Introducción}

Se encuentra, que se utilizan de manera indistinta los términos "prácticas pedagógicas, prácticas de enseñanza y prácticas docentes". Junto a ello, Zuluaga, (2007) indica que [...] "No pocos historiadores de la educación y la pedagogía sitúan, a partir de la Didáctica magna de Comenio, el nacimiento de una disciplina autónoma que unos denominan pedagogía, otros ciencia de la educación, otros didáctica y otros ciencias de la educación" [...] (p.100). Así, resulta abrumador hablar de las prácticas que utiliza el docente para la enseñanza, pues para algunos investigadores, en el campo de las matemáticas es correcto hablar de didáctica de la enseñanza, pero errado hablar de prácticas pedagógicas, debido a que la pedagogía se ocupa de otros asuntos, pero se define la pedagogía como "la ciencia que se ocupa de la educación y la enseñanza, cuyo objetivo es proporcionar guías para planificar, ejecutar y evaluar procesos de enseñanza y aprendizaje" ${ }^{3}$, aunque el fin último de la actividad docente es la enseñanza.

\section{Problema de investigación}

En sus variadas prácticas, el trabajo que enfrentan los docentes en la enseñanza de las fracciones y el tratamiento de situaciones problema es complejo, ello tiene que ver con la dificultad marcada en este tema, el cual se enseña en los grados $4^{\circ}, 5^{\circ}, 6^{\circ}$ y $7^{\circ}$ de la educación básica, pero en grados posteriores, cuando se necesita que apliquen operaciones con fracciones, para resolver problemas asociados al tema, para muchos estudiantes se convierte en un obstáculo.

Pregunta de Investigación. ¿En que medida, según el tipo de práctica de enseñanza empleada por los docentes se favorecen la comprensión, los procesos de aprendizaje y las destrezas de los estudiantes, en cuanto a la resolución de situaciones problema?

\section{Contexto de la investigación}

Está delimitado por tres grupos de grado séptimo $\left(7^{\circ}\right)$ de una Institución Educativa de carácter público, del municipio de Fredonia, departamento de Antioquia, donde se brinda educación formal a una población flotante que circunda los 1048 educandos en los niveles de básica primaria, secundaria y media, provenientes de los niveles económicos 1 y 2 , pertenecientes a grupos étnicos variados, en

4 Diccionario de la real academia de la lengua española 
donde sobresale el mestizaje. La economía de la región depende de la agricultura, donde sobresale el comercio del café.

\section{Objetivo general}

Caracterizar y analizar las prácticas de enseñanza de algunos docentes de Matemáticas de grado séptimo, para relacionar y categorizar tales prácticas en concordancia con las destrezas que desarrollan sus estudiantes frente a la resolución de problemas, detectando desde la exploración y el análisis de las prácticas docentes, factores praxeológicos y epistemológicos asociados a la capacidad de los estudiantes para solucionar problemas en contexto y los procesos que se llevan a cabo para la resolución de problemas apoyados en las fracciones.

\section{Objetivos especificos}

1. Interpretar desde las prácticas pedagógicas de los docentes, los factores que inciden o están asociados a la capacidad de los estudiantes para resolver problemas en contexto.

2. Evaluar hasta que punto tales prácticas les permiten apropiarse de herramientas que transformen sus destrezas y eficacia para resolver problemas con fracciones.

3. Caracterizar las prácticas de enseñanza que utilizan los docentes de matemáticas séptimo grado, para asociarlas con la generación de destrezas en la resolución de problemas por parte de los estudiantes, desde el escenario de las fracciones.

\section{Justificación}

El estudio de las prácticas de enseñanza de los docentes posibilita evidenciar cuales permiten que el estudiante se motive mas y cuales mejoran o generan más habilidades; ello se justifica en tanto se encuentren elementos que favorezcan la adquisición de habilidades y se mejore el proceso de razonamiento en los estudiantes, para solucionar problemas en contexto, y determinar aspectos que permitan establecer conexiones en el proceso de enseñanza, que propicien a su vez mejoras en los desempeños en resolución de problemas en contexto, lo cual de manera colateral, permitirá elevar los niveles de calidad en la institución educativa, y que el porcentaje de acceso a la educación superior sea más significativo; adicionalmente esperar mejores desempeños en resultados de pruebas externas, a nivel Municipal y Departamental.

\section{Marco teórico de la investigación}

Se va a definir un Marco Teórico centrado en las Prácticas de enseñanza de docentes de matemáticas, con escenario para su desarrollo en la resolución de problemas con fracciones, y el abordaje del marco mencionado se hará bajo los siguientes componentes:

- Breve historia de la Educación, centrada en las distintas formas de enseñanza aplicadas, de acuerdo con los modelos de cada época, los mismos que están influenciados por distintos factores, tales como: religión, poder económico; poder ideológico, etc.

- Recorrido histórico de las practicas de enseñanza, de acuerdo con particularidades de cada época, enfocando las mismas hacia las practicas constructivas

- Revisión a cerca de las teorías del aprendizaje y los cambios en la enseñanza de las matemáticas

- Principales exponentes históricos de la heurística como método utilizado para el abordaje de la resolución de problemas, y la percepción actual,

- Resumen de aspectos históricos del uso de la fracción en las diferentes culturas antiguas, $y$ su evolución con el paso del tiempo

- Aspectos históricos y referencias del proceso de enseñanza - aprendizaje de las fracciones. 
El marco teórico en esta investigación será el de prácticas de enseñanza en matemáticas, focalizado en el proceso de resolución de problemas, desde el concepto de fracciones, en particular los que involucran particiones, razones y porcentajes pertinentes para grado séptimo. La fundamentación teórica que se propone en este trabajo de investigación, expone algunas teorías en cuanto a las prácticas de enseñanza a través de la historia embocándolas hacia las enunciadas por Mondragón (2004) especialmente las Constructivas. Frente a la resolución de problemas en matemáticas, se retoman aportes de Polya, Schoenfeld y Santos Trigo.

\section{Diversidad de las prácticas de enseñanza}

Según Partido (2003) los factores que inducen al docente a seleccionar un contenido $u$ otro, una estrategia de aprendizaje $u$ otra, pueden ser fundamentalmente de dos tipos: los factores internos, es decir, los propios del docente; y los factores externos al docente: los estudiantes, la situación de clase, los padres de familia o las autoridades educativas. De acuerdo con Mondragón (2004), entre las prácticas más comunes que utilizan los docentes tenemos: expositivas; de profundización; lúdicas y constructivas.

Practicas constructivas. En concordancia con el principal interés de la propuesta de investigación, el centro de atención serán las prácticas constructivas, que se equiparan con el modelo constructivista, tomando junto a ellas, la resolución de problemas como proceso matemático, y las fracciones como el concepto matemático.

\section{La heurística en la enseñanza de las matemáticas}

La heurística es la actividad asociada a la implementación de técnicas para resolver problemas, desde un aspecto práctico o informal. Ella enuncia ciertos procedimientos en cuanto a descubrir formas, herramientas y métodos accesibles a los estudiantes que les permitan solucionar problemas de su entorno cotidiano. El reconocimiento que se le ha dado a la actividad de resolver problemas en el desarrollo de las matemáticas ha originado propuestas sobre su enseñanza, entre las cuales tenemos las de investigadores (Polya, 1962; Schoenfeld, 1985; Santos Trigo, 1996; Valero, 2002; Vila \& Callejo, 2005; Obando \& Múnera, 2003; Nieto, 2004; MEN, 1998; 2006)

En cuanto al concepto "resolución", encontramos una variada terminología asociada a ella, entre los que se cuentan: resolución de problemas, resolución de situaciones problema, resolución de situaciones problémicas, resolución de situaciones problemáticas y resolución de situaciones problematizadoras. Son variadas las posturas de autores y publicaciones al respecto, así, en $\mathrm{MEN}^{3}$ (2006), se habla de situaciones problema (pp. 49,52,69,71,74,77); en MEN (1998) se habla tanto de situaciones problemáticas (pp.15,16,18,19,24), de situaciones problémicas (p.54), como de situaciones problematizadoras (pp. $17,50,89)$.

\section{Metodología}

Este trabajo de investigación se realiza mediante un enfoque cualitativo, dado que su carácter inductivo permite que el investigador, desarrolle conceptos y comprensiones partiendo de los datos encontrados en el escenario, en tal sentido, la pretensión no es generalizar los hallazgos, sino describir y comprender como las prácticas de enseñanza de los docentes se convierten en herramientas que permiten a los estudiantes en el aula, generar procesos de aprendizaje, en relación con la resolución de problemas, mediados por fracciones.

Tipo de estudio. De acuerdo con la investigación, a partir de la cantidad de unidades de análisis seleccionadas como casos, (tres profesoras y nueve estudiantes) de una clase de matemáticas de grado séptimo, el tipo de estudio, dado que cada uno de los docentes y estudiantes presentan características particulares, obedece a un "estudio colectivo de caso" Stake, (1999) ya que posibilita indagar un fenómeno, población o condición general a partir del estudio intensivo de varios casos (p.17).

\footnotetext{
3 Ministerio de Educación Nacional. (2006). Estándares Básicos de Competencias. Bogotá.

4 Ministerio de Educación Nacional. (1998). Lineamientos curriculares de matemáticas. Santafe de Bogota.
} 


\section{Participantes}

Se seleccionan los estudiantes intencionalmente, previo criterio teórico, en donde se tiene en cuenta el concepto de las tres docentes involucradas, a las cuales, por su conocimiento del grupo, se les solicita escoger un estudiante que se caracterice por presentar habilidades marcadas para trabajar en matemáticas y otro que se caracterice por presentar dificultades marcadas en esta área.

\section{Fuentes de recolección de datos}

Los datos necesarios para la realización del informe respecto del análisis de la información, se recolectan a través de fuentes como: observación directa, videograbaciones, entrevistas semi-estructuradas, documentos escritos (diarios de campo del docente y pruebas escritas), de lo cual a continuación se da una breve descripción:

\section{Actividades: fases del trabajo de campo}

El trabajo de campo para el desarrollo de la investigación se realiza en tres fases:

Fase1. Observación de clases a las docentes, registro de la información y realización de entrevistas. Fase inicial, en donde se realizaron conversatorios con tres docentes de matemáticas, en cuanto a la posibilidad de observar sus prácticas de enseñanza; tomando algunos estudiantes, para indagar, como ellas ofrecen facilidades en cuanto a la resolución de problemas.
Fase 2. Realización de entrevista preliminar a los estudiantes. Se plantearon situaciones relacionadas con el trabajo en el aula de clases y, que permitan extraer o descubrir variables respecto de la resolución de situaciones problema, y el proceso algorítmico de las mismas.

Fase 3. Solución de guías y entrevistas posteriores. Aquí se elaboran y aplican una: Prueba diagnóstica; Prueba intermedia y Prueba final. Una vez aplicadas las tres guías, se analiza la información para observar particularidades en los procesos de resolución.

\section{Referencias}

Zuluaga, Garcés, O. L. La Didáctica Magna. Comenio, J. Tomado de: Otra vez Comenio publicado en Revista Educación y pedagogía, Vol.19, Nº. 47, Enero-Abril de 2007. pp. 99-100.

Mondragón, Ochoa, H. (2004). Prácticas pedagógicas en la universidad para la construcción de ambientes de aprendizaje significativo. Universidad Javeriana de Cali. Colombia.

Partido Calva, M. (2003). Concepciones y estrategias didácticas sobre la lectura. Colección pedagógica universitaria, número 39. EneroJunio, 2003.

Stake, R. E. (1999). Investigación con estudio de casos. Segunda edición. Ediciones Morata. Madrid. Título original de la obra: the art of case study research. 1995 by sage publications, inc. 